\title{
VERTEX OPERATORS FOR ADS3 WITH RAMOND BACKGROUND
}

\author{
L. DOLAN \\ Department of Physics and Astronomy, University of North Carolina \\ Chapel HIll, NC 27599-3255, USA
}

\begin{abstract}
This review gives results on vertex operators for the Type IIB superstring in an AdS3 $\mathrm{x}$ S3 background with Ramond-Ramond flux, which were presented at Strings 2000. Constraint equations for these vertex operators are derived, and their components are shown to satisfy the supergravity linearized equations of motion for the six-dimensional $(2,0)$ theory of a supergravity and tensor multiplet expanded around AdS3 x S3 spacetime.
\end{abstract}

\section{Introduction}

The conjectured duality between M-theory or Type IIB string theory on anti-de Sitter (AdS) space and the conformal field theory on the boundary of AdS space may be useful in giving a controlled systematic approximation for strongly coupled gauge theories. Examples with maximal supersymmetry correspond to a set of $p$ branes whose near horizon geometry looks like $A d S_{p+2} \times S^{\mathcal{D}-p-2}$, where $\mathcal{D}=10$ or 11 for branes in string or M-theory, and $p=2,3,5$. The formulation of vertex operators and string theory tree amplitudes for the IIB superstring on $A d S_{5} \times S^{5}$ will allow access to the dual conformal $S U(N)$ gauge field theory $C F T_{4}$ at large $N$, but small fixed 't Hooft coupling $x=g_{Y M}^{2} N$ in the dual picture, as $\left(g_{Y M}^{2} N\right)^{\frac{1}{2}}(4 \pi)^{\frac{1}{2}}=$ $R_{s p h}^{2} / \alpha^{\prime}$. Presently only the large $N$, and large fixed 't Hooft coupling $x$ limit is accessible in the $C F T$, since only the supergravity limit $\left(\alpha^{\prime} \rightarrow 0\right)$ of the correlation functions of the AdS theory is known.

In this talk we discuss a case with non-maximal supersymmetry that is related to a system with a $D 1$-brane and a $D 5$-brane. Its quantizable worldsheet action ${ }^{1}$ describes the IIB string on $A d S_{3} \times S^{3} \times M$ with background Ramond-Ramond flux, where $M$ is $T^{4}$ or $K 3$. The vertex operators for this model can be computed explicitly in the bulk. ${ }^{2}$ Correlation functions constructed from these vertex operators, restricted to the boundary of $A d S_{3}$, would be those for a two-dimensional spacetime conformal field theory. We work to leading order in $\alpha^{\prime}$, but because of the high degree of symmetry of the model, we expect our result for the vertex oper- 
ators to be exact. Tree level $n$-point correlation functions for $n \geq 4$ presumably have $\alpha^{\prime}$ corrections, since the worldsheet theory is not a free conformal field theory. But there may be sufficiently many symmetry currents to determine the tree level correlation functions exactly in $\alpha^{\prime}$ as well. ${ }^{3}$

In terms of Berkovits-Vafa-Witten worldsheet variables, constraint equations for the vertex operators in the flat space $\mathcal{R}^{6}$ follow from the physical state conditions coming from an $N=4$ superconformal algebra. We generalize ${ }^{2}$ these constraint equations to $A d S_{3} \times S^{3}$ for the vertex operators of the massless states that are independent of the compactification $M$. We then solve the constraints and identify the components of the vertex operators as supergravity fields, that satisfy the $D=6$, $N=(2,0)$ theory ${ }^{4}$ linearized around the $A d S_{3} \times S^{3}$ background.

Recent work ${ }^{5,6}$ discusses covariant ten-dimensional worldsheet variables and extends our analysis to vertex operators on $A d S_{5}$ in a spinor formulation.

\section{Formulating Strings on $A d S$}

In the Ramond-Neveu-Schwarz (RNS) formalism, the worldsheet action for strings on $A d S$ space with background Ramond-Ramond flux involves $2 \mathrm{~d}$ spin fields. These violate superconformal worldsheet symmetry, since the worldsheet supercurrents are not local with respect to the spin fields, and their presence makes the worldsheet theory difficult to understand.

For the Type IIB superstring on $A d S_{3} \times S^{3}$ case, a sigma model ${ }^{1}$ with conventional local interactions (no spin fields in the action) was found using the supergroup $P S U(2 \mid 2)$ as target, coupled to ghost fields $\rho$ and $\sigma$. The spacetime symmetry group is $P S U(2 \mid 2) \times P S U(2 \mid 2)$, acting by left and right multiplication on the group manifold, i.e. by $g \rightarrow a g b^{-1}$ where $g$ is a $P S U(2 \mid 2)$-valued field, and $a, b \in P S U(2 \mid 2)$ are the symmetry group's Lie algebra elements. The supergroup is generated by the super Lie algebra with 12 bosonic generators forming a subalgebra $S O(4) \times S O(4)$ together with 16 odd generators. Hence our model has non-maximal supersymmetry with 16 supercharges.

The worldsheet field content generalizes the Berkovits-Vafa formalism which provides a manifest Lorentz covariant and supersymmetric quantization on $R^{6}$. Its six bosonic fields $x^{p}(z, \bar{z})$ contain both left- and right-moving modes. In addition there are left-moving fermi fields $\theta_{L}^{a}(z), p_{L}^{a}(z)$ of spins 0 and 1 , together with ghosts $\sigma_{L}(z), \rho_{L}(z)$, and right-moving counterparts of all these left-moving fields. These variables allow Ramond-Ramond background fields to be incorporated without adding spin fields to the worldsheet action as follows: in the $A d S_{3} \times S^{3}$ case, i.e. after adding RR background fields to the worldsheet action, one can integrate out the $p$ 's, so that the model has ordinary conformal fields $x^{p}, \theta^{a}, \bar{\theta}^{a}$ (all now with both left- and right-moving components) as well as the ghosts. The $P S U(2 \mid 2)$ valued field $g$ is given in terms of $x, \theta$, and $\bar{\theta}$, which are identified as coordinates on the supergroup manifold. In addition, Type IIB on $A d S_{3} \times S^{3} \times M$ has worldsheet variables describing the compactification on the four-dimensional space $M$. Their Virasoro currents have central charge $c=6$, and will be labeled with a subscript $C$. 


\section{3. $N=4$ Super Virasoro Generators}

The holomorphic $N=4$ superconformal generators with $c=6$ are given for flat space by ${ }^{1}$

$$
\begin{aligned}
T= & -\frac{1}{2} \partial x^{m} \partial x_{m}-p_{a} \partial \theta^{a}-\frac{1}{2} \partial \rho \partial \rho-\frac{1}{2} \partial \sigma \partial \sigma+\partial^{2}(\rho+i \sigma)+T_{C} \\
G^{+}= & -e^{-2 \rho-i \sigma}(p)^{4}+\frac{i}{2} e^{-\rho} p_{a} p_{b} \partial x^{a b} \\
& +e^{i \sigma}\left(-\frac{1}{2} \partial x^{m} \partial x_{m}-p_{a} \partial \theta^{a}-\frac{1}{2} \partial(\rho+i \sigma) \partial(\rho+i \sigma)+\frac{1}{2} \partial^{2}(\rho+i \sigma)\right)+G_{C}^{+} \\
G^{-}= & e^{-i \sigma}+G_{C}^{-} \\
J= & \partial(\rho+i \sigma)+J_{C} \\
\tilde{G}^{+}= & e^{i H_{C}}\left[-e^{-3 \rho-2 i \sigma}(p)^{4}+\frac{i}{2} e^{-2 \rho-i \sigma} p_{a} p_{b} \partial x^{a b}\right. \\
& +e^{-\rho}\left(-\frac{1}{2} \partial x^{m} \partial x_{m}-p_{a} \partial \theta^{a}-\frac{1}{2} \partial(\rho+i \sigma) \partial(\rho+i \sigma)\right. \\
& \left.+\frac{1}{2} \partial^{2}(\rho+i \sigma)\right]+e^{-\rho-i \sigma} \tilde{G}_{C}^{-} \\
J^{+}= & e^{\rho+i \sigma} J_{C}^{+} \\
J^{-}= & e^{-\rho-i \sigma} J_{C}^{-} .
\end{aligned}
$$

These currents are given in terms of the left-moving bosons $\partial x^{m}, \rho, \sigma$, and the leftmoving fermionic worldsheet fields $p^{a}, \theta^{a}$, where $1 \leq m \leq 6,1 \leq a \leq 4$. There are corresponding anti-holomorphic expressions. Both sets of generators are used to implement the physical state conditions on the vertex operators, a procedure which results in a set of string constraint equations.

\section{String Constraint Equations for the Vertex Operators}

The expansion of the massless vertex operator in terms of the worldsheet fields is

$$
V=\sum_{m, n=-\infty}^{\infty} e^{m(i \sigma+\rho)+n(i \bar{\sigma}+\bar{\rho})} V_{m, n}(x, \theta, \bar{\theta}) .
$$

In flat space, the constraints from the left and right-moving worldsheet super Virasoro algebras are:

$$
\begin{aligned}
(\nabla)^{4} V_{1, n} & =\nabla_{a} \nabla_{b} \partial^{a b} V_{1, n}=0 \\
\frac{1}{6} \epsilon^{a b c d} \nabla_{b} \nabla_{c} \nabla_{d} V_{1, n} & =-i \nabla_{b} \partial^{a b} V_{0, n} \\
\nabla_{a} \nabla_{b} V_{0, n}-\frac{i}{2} \epsilon_{a b c d} \partial^{c d} V_{-1, n} & =0, \quad \nabla_{a} V_{-1, n}=0 ; \\
\bar{\nabla}^{4} V_{n, 1} & =\bar{\nabla}_{\bar{a}} \bar{\nabla}_{\bar{b}} \bar{\partial}^{\bar{a} \bar{b}} V_{n, 1}=0 \\
\frac{1}{6} \epsilon^{\bar{a} \bar{c} \bar{c} \bar{d}} \bar{\nabla}_{\bar{b}} \bar{\nabla}_{\bar{c}} \bar{\nabla}_{\bar{d}} V_{n, 1} & =-i \bar{\nabla}_{\bar{b}} \bar{\partial}^{\bar{a} \bar{b}} V_{n, 0} \\
\bar{\nabla}_{\bar{a}} \bar{\nabla}_{\bar{b}} V_{n, 0}-\frac{i}{2} \bar{\epsilon}_{\bar{a} \bar{b} \bar{c} \bar{d}} \bar{\partial}^{\bar{c} \bar{d}} V_{n,-1} & =0, \quad \bar{\nabla}_{\bar{a}} V_{n,-1}=0 \\
\partial^{p} \partial_{p} V_{m, n} & =0
\end{aligned}
$$

for $-1 \leq m, n \leq 1$, with the notation $\nabla_{a}=d / d \theta^{a}, \bar{\nabla}_{\bar{a}}=d / d \bar{\theta}^{\bar{a}}, \partial^{a b}=-\sigma^{p a b} \partial_{p}$. These equations were derived in flat space by requiring the vertex operators to 
satisfy the physical state conditions

$$
\begin{array}{r}
G_{0}^{-} V=\tilde{G}_{0}^{-} V=\bar{G}_{0}^{-} V=\overline{\tilde{G}}_{0}^{-} V=T_{0} V=\bar{T}_{0} V=0 \\
J_{0} V=\bar{J}_{0} V=0, \quad G_{0}^{+} \tilde{G}_{0}^{+} V=\bar{G}_{0}^{+} \overline{\tilde{G}}_{0}^{+} V=0
\end{array}
$$

where $T_{n}, G_{n}^{ \pm}, \tilde{G}_{n}^{ \pm}, J_{n}, J_{n}^{ \pm}$and corresponding barred generators are the left and right $N=4$ worldsheet superconformal generators. These conditions further imply $V_{m, n}=0$ for $m>1$ or $n>1$ or $m<1$ or $n<1$, leaving nine non-zero components.

In $A d S_{3} \times S^{3}$ space, we generalize these equations as follows ${ }^{2}$ :

$$
\begin{aligned}
F^{4} V_{1, n} & =F_{a} F_{b} K^{a b} V_{1, n}=0 \\
\frac{1}{6} \epsilon^{a b c d} F_{b} F_{c} F_{d} V_{1, n} & =-i F_{b} K^{a b} V_{0, n}+2 i F^{a} V_{0, n}-E^{a} V_{-1, n} \\
F_{a} F_{b} V_{0, n}-\frac{i}{2} \epsilon_{a b c d} K^{c d} V_{-1, n} & =0, \quad F_{a} V_{-1, n}=0 ; \\
\bar{F}^{4} V_{n, 1} & =\bar{F}_{\bar{a}} \bar{F}_{\bar{b}} \bar{K}^{\bar{a} \bar{b}} V_{n, 1}=0 \\
\frac{1}{6} \epsilon^{\bar{a} \bar{b} \bar{c} \bar{d}} \bar{F}_{\bar{b}} \bar{F}_{\bar{c}} \bar{F}_{\bar{d}} V_{n, 1} & =-i \bar{F}_{\bar{b}} \bar{K}^{\bar{a} \bar{b}} V_{n, 0}+2 i \bar{F}^{\bar{a}} V_{n, 0}-\bar{E}^{\bar{a}} V_{n,-1} \\
\bar{F}_{\bar{a}} \bar{F}_{\bar{b}} V_{n, 0}-\frac{i}{2} \bar{\epsilon}_{\bar{a} \bar{b} \bar{c} \bar{d}} \bar{K}^{\bar{c} \bar{d}} V_{n,-1} & =0, \quad \bar{F}_{\bar{a}} V_{n,-1}=0 .
\end{aligned}
$$

There is also a spin zero condition constructed from the Laplacian

$$
\left(F_{a} E_{a}+\frac{1}{8} \epsilon_{a b c d} K^{a b} K^{c d}\right) V_{n, m}=\left(\bar{F}_{\bar{a}} \bar{E}_{\bar{a}}+\frac{1}{8} \bar{\epsilon}_{\bar{a} \bar{b} \bar{c} \bar{d}} \bar{K}^{\bar{a} \bar{b}} K^{\bar{c} \bar{d}}\right) V_{n, m}=0 .
$$

We derived ${ }^{2}$ the curved space equations (7-9) by deforming the equations for the flat case (3-5), by requiring invariance under the $P S U(2 \mid 2)$ transformations (10) that replace the $D=6$ super Poincare transformations of flat space. The Lie algebra of the supergroup $P S U(2 \mid 2)$ contains six even elements $K_{a b} \in S O(4)$ and eight odd ones $E_{a}, F_{a}$. They generate the infinitesimal symmetry transformations of the constraint equations:

$$
\begin{aligned}
\Delta_{a}^{-} V_{m, n} & =F_{a} V_{m, n}, \quad \Delta_{a b} V_{m, n}=K_{a b} V_{m, n} \\
\Delta_{a}^{+} V_{1, n}=E_{a} V_{1, n}, \quad \Delta_{a}^{+} V_{0, n} & =E_{a} V_{0, n}+i F_{a} V_{1, n}, \quad \Delta_{a}^{+} V_{-1, n}=E_{a} V_{-1, n}-i F_{a} V_{0, n} .
\end{aligned}
$$

We write $E_{a}, F_{a}$, and $K_{a b}$ for the operators that represent the left action of $e_{a}, f_{a}$, and $t_{a b}$ on $g$. In the above coordinates,

$$
\begin{aligned}
F_{a} & =\frac{d}{d \theta^{a}}, \quad K_{a b}=-\theta_{a} \frac{d}{d \theta^{b}}+\theta_{b} \frac{d}{d \theta^{a}}+t_{L a b} \\
E_{a} & =\frac{1}{2} \epsilon_{a b c d} \theta^{b}\left(t_{L}^{c d}-\theta^{c} \frac{d}{d \theta_{d}}\right)+h_{a \bar{b}} \frac{d}{d \bar{\theta}_{\bar{b}}},
\end{aligned}
$$


where we have introduced an operator $t_{L}$ that generates the left action of $S U(2) \times$ $S U(2)$ on $h$ alone, without acting on the $\theta$ 's. Here

$$
\begin{gathered}
g=g(x, \theta, \bar{\theta})=e^{\theta^{a} f_{a}} e^{\frac{1}{2} \sigma^{p c d} x_{p} t_{c d}} e^{\bar{\theta}^{\bar{a}} e_{\bar{a}}}=e^{\theta^{a} f_{a}} h(x) e^{\bar{\theta}^{\bar{a}} e_{\bar{a}}}, \\
t_{L a b} g=e^{\theta^{a} f_{a}}\left(-t_{a b}\right) h(x) e^{\bar{\theta}^{\bar{a}} e_{\bar{a}}}
\end{gathered}
$$

and we found (11) by requiring $F_{a} g=f_{a} g, E_{a} g=e_{a} g, K_{a b} g=-t_{a b} g$. Similar expressions $^{2}$ hold for the right-acting generators $\bar{K}_{\bar{a} \bar{b}}, \bar{E}_{\bar{a}}$, and $\bar{F}_{\bar{a}}$.

\section{Supersymmetry Algebras}

In flat space, the $D=6$ supersymmetry algebra for the left-movers is given by

$$
\begin{aligned}
\left\{q_{a}^{+}, q_{c}^{-}\right\} & =\frac{1}{2} \epsilon_{a b c d} P^{c d} \\
{\left[P_{a b}, P_{c d}\right] } & =0=\left[P_{a b}, q_{c}^{ \pm}\right]=\left\{q_{a}^{+}, q_{b}^{+}\right\}=\left\{q_{a}^{-}, q_{b}^{-}\right\}
\end{aligned}
$$

where $P_{a b} \equiv \delta_{a c} \delta_{b d} P^{c d}$ and

$$
\begin{aligned}
q_{a}^{-} & =\oint F_{a}(z) \\
q_{a}^{+} & =\oint\left(e^{-\rho-i \sigma} F_{a}(z)+i E_{a}(z)\right) \\
P^{a b} & =\oint \partial x_{m}(z) \sigma^{m a b} .
\end{aligned}
$$

In flat space we have $F_{a}(z)=p_{a}(z)$ and $E_{a}(z)=\frac{1}{2} \epsilon_{a b c d} \theta^{b}(z) \partial x_{m}(z) \sigma^{m c d}$. We distinguish between the currents and their zero moments $E_{a}, F_{a}$ which together with $P_{a b}$ also generate the flat space supersymmetry algebra

$$
\begin{aligned}
{\left[P_{a b}, P_{c d}\right] } & =0=\left[P_{a b}, F_{c}\right]=\left[P_{a b}, E_{c}\right], \\
\left\{E_{a}, F_{b}\right\} & =\frac{1}{2} \epsilon_{a b c d} P^{c d}, \quad\left\{E_{a}, E_{b}\right\}=\left\{F_{a}, F_{b}\right\}=0 .
\end{aligned}
$$

On $A d S_{3} \times S^{3}$, the Poincare supersymmetry algebra (17) is replaced by the $P S U(2 \mid 2)$ superalgebra

$$
\begin{aligned}
{\left[K_{a b}, K_{c d}\right] } & =\delta_{a c} K_{b d}-\delta_{a d} K_{b c}-\delta_{b c} K_{a d}+\delta_{b d} K_{a c} \\
{\left[K_{a b}, E_{c}\right] } & =\delta_{a c} E_{b}-\delta_{b c} E_{a}, \quad\left[K_{a b}, F_{c}\right]=\delta_{a c} F_{b}-\delta_{b c} F_{a} \\
\left\{E_{a}, F_{b}\right\} & =\frac{1}{2} \epsilon_{a b c d} K^{c d}, \quad\left\{E_{a}, E_{b}\right\}=0=\left\{F_{a}, F_{b}\right\} .
\end{aligned}
$$

The generators $q_{a}^{ \pm}$, which generate the $A d S$ tranformations (10), still have a form similar to (15) but $E_{a}(z, \bar{z}), F_{a}(z, \bar{z}), K_{a b}(z, \bar{z})$ are no longer holomorphic and their zero moments with respect to $z$ satisfy (18). 


\section{String Equations for the $A d S$ Vertex Operator Components}

The $A d S$ supersymmetric constraints (7-9) imply ${ }^{2}$

$$
\begin{gathered}
F_{a} F_{b} K^{a b} V_{1,1}=0, \quad \bar{F}_{\bar{a}} \bar{F}_{\bar{b}} \bar{K}^{\bar{a} \bar{b}} V_{1,1} \\
\left(F_{a} E_{a}+\frac{1}{8} \epsilon_{a b c d} K^{a b} K^{c d}\right) V_{1,1}=\left(\bar{F}_{\bar{a}} \bar{E}_{\bar{a}}+\frac{1}{8} \bar{\epsilon}_{\bar{a} \bar{b} \bar{c} \bar{d}} \bar{K}^{\bar{a} \bar{b}} K^{\bar{c} \bar{d}}\right) V_{1,1}=0 .
\end{gathered}
$$

The vertex operators $V_{-1,1}, V_{1,-1}, V_{0,-1}, V_{-1,0}, V_{-1,-1}$ can be gauge fixed to zero, and therefore do not correspond to propagating degrees of freedom. Furthermore, this gauge symmetry can be used both to set to zero the components of $V_{1,1}$ with no $\theta$ 's or no $\bar{\theta}$ 's, and to gauge fix all components of $V_{0,1}, V_{1,0}, V_{0,0}$ that are independent of those of $V_{1,1}$. The physical degrees of freedom of the massless compactification independent vertex operators are thus described by a superfield

$$
\begin{aligned}
V_{1,1}= & \theta^{a} \bar{\theta}^{\bar{a}} V_{a \bar{a}}^{--}+\theta^{a} \theta^{b} \bar{\theta}^{\bar{a}} \sigma_{a b}^{m} \bar{\xi}_{m \bar{a}}^{-}+\theta^{a} \bar{\theta}^{\bar{a}} \bar{\theta}^{\bar{b}} \sigma_{\bar{a} \bar{b}}^{m} \xi_{m a}^{-} \\
& +\theta^{a} \theta^{b} \bar{\theta}^{\bar{a}} \bar{\theta}^{\bar{b}} \sigma_{a b}^{m} \sigma_{\bar{a} \bar{b}}^{n}\left(g_{m n}+b_{m n}+\bar{g}_{m n} \phi\right)+\theta^{a}\left(\bar{\theta}^{3}\right)_{\bar{a}} A_{a}^{-+\bar{a}}+\left(\theta^{3}\right)_{a} \bar{\theta}^{\bar{a}} A_{\bar{a}}^{+-a} \\
& +\theta^{a} \theta^{b}\left(\bar{\theta}^{3}\right)_{\bar{a}} \sigma_{a b}^{m} \bar{\chi}_{m}^{+\bar{a}}+\left(\theta^{3}\right)^{a} \bar{\theta}^{\bar{a}} \bar{\theta}^{\bar{b}} \sigma_{\bar{a} \bar{b}}^{m} \chi_{m}^{+a}+\left(\theta^{3}\right)_{a}\left(\bar{\theta}^{3}\right)_{\bar{a}} F^{++a \bar{a}}
\end{aligned}
$$

This has the field content of $D=6, N=(2,0)$ supergravity with one supergravity and one tensor multiplet. Further massless multiplets correspond to the compactification degrees of freedom. In flat space, the surviving constraint equations imply that the component fields $\Phi$ are all on shell massless fields, that is $\sum_{m=1}^{6} \partial^{m} \partial_{m} \Phi=0$ as in (5), and in addition

$$
\begin{aligned}
\partial^{m} g_{m n} & =-\partial_{n} \phi, \quad \partial^{m} b_{m n}=0, \quad \partial^{m} \chi_{m}^{ \pm b}=\partial^{m} \bar{\chi}_{m}^{ \pm \bar{b}}=0 \\
\partial_{a b} \chi_{m}^{ \pm b} & =\partial_{\bar{a} \bar{b}} \chi_{m}^{ \pm \bar{b}}=0, \quad \partial_{c b} F^{ \pm \pm b \bar{a}}=\partial_{\bar{c} \bar{b}} F^{ \pm \pm \bar{b} a}=0,
\end{aligned}
$$

where

$$
\begin{aligned}
& F^{+-a \bar{a}}=\partial^{\bar{a} \bar{b}} A_{\bar{b}}^{+-a}, \quad F^{-+a \bar{a}}=\partial^{a b} A_{b}^{-+\bar{a}}, \quad F^{--a \bar{a}}=\partial^{a b} \partial^{\bar{a} \bar{b}} V_{b \bar{b}}^{--} \\
& \chi_{m}^{-a}=\partial^{a b} \xi_{m b}^{-}, \quad \bar{\chi}_{m}^{-\bar{a}}=\partial^{\bar{a} \bar{b}} \bar{\xi}_{m \bar{b}}^{-} .
\end{aligned}
$$

The equations of motion for the flat space vertex operator component fields describe $D=6, N=(2,0)$ supergravity $^{4}$ expanded around the six-dimensional Minkowski metric.

In $A d S_{3} \times S^{3}$ space corresponding gauge transformations reduce the number of degrees of freedom in a similar fashion, but the Laplacian must be replaced by the $A d S$ Laplacian, and the constraints are likewise deformed. We focus on the vertex operator $V_{11}$ that carries the physical degrees of freedom. We show the string constraint equations are equivalent to the $D=6, N=(2,0)$ linearized supergravity equations expanded around the $A d S_{3} \times S^{3}$ metric.

For the bosonic field components of the vertex operator the $A d S$ constraint equations result in 


$$
\begin{gathered}
\square h_{\bar{a}}^{g} V_{a g}^{--}=-4 \sigma_{a b}^{m} \sigma_{g h}^{n} \delta^{b h} h_{\bar{a}}^{g} G_{m n} \\
\square h_{\bar{a}}^{g} h_{\bar{b}}^{h} \sigma_{a b}^{m} \sigma_{g h}^{n} G_{m n}=\frac{1}{4} \epsilon_{a b c e} \epsilon_{f g h k} \delta^{c h} h_{\bar{a}}^{f} h_{\bar{b}}^{g} F^{++e k} \\
\square h_{g}^{\bar{a}} F^{++a g}=0, \quad \square h_{g}^{\bar{a}} A_{a}^{-+g}=0, \quad \square h_{\bar{a}}^{g} A_{g}^{+-a}=0 \\
\epsilon_{e a c d} t_{L}^{c d} h_{\bar{a}}^{b} A_{b}^{+-a}=0, \quad \epsilon_{\bar{e} \bar{b} \bar{c} \bar{d}} t_{R}^{\bar{c} \bar{d}} h_{a}^{\bar{a}} A_{\bar{a}}^{-+\bar{b}}=0 \\
\epsilon_{e a c d} t_{L}^{c d} h_{b}^{\bar{a}} F^{++a b}=0, \quad \epsilon_{\bar{e} \bar{b} \bar{c} \bar{d}} t_{R}^{\bar{c} \bar{d}} h_{\bar{a}}^{a} F^{++\bar{a} \bar{b}}=0 \\
t_{L}^{a b} h_{\bar{a}}^{g} h_{\bar{b}}^{h} \sigma_{a b}^{m} \sigma_{g h}^{n} G_{m n}=0, \quad t_{R}^{\bar{a} \bar{b}} h_{a}^{\bar{g}} h_{b}^{\bar{h}} \sigma_{\bar{g} \bar{h}}^{m} \sigma_{\bar{a} \bar{b}}^{n} G_{m n}=0 .
\end{gathered}
$$

We have expanded $G_{m n}=g_{m n}+b_{m n}+\bar{g}_{m n} \phi$. The $S O(4)$ Laplacian is $\square \equiv \frac{1}{8} \epsilon_{a b c d} t_{L}^{a b} t_{L}^{c d}=\frac{1}{8} \epsilon_{\bar{a} \bar{b} \bar{c} \bar{d}} t_{R}^{\bar{a} \bar{b}} t_{R}^{\bar{c} \bar{d}}$. In order to compare this with supergravity, we need to reexpress the above formulas containing the right- and left-invariant vielbeins $t_{L}^{a b}, t_{R}^{\bar{a} \bar{b}}$ in terms of covariant derivatives $D_{p}$ on the group manifold. So we write

$$
\mathcal{T}_{L}^{c d} \equiv-\sigma^{p c d} D_{p}, \quad \mathcal{T}_{R}^{\bar{c} \bar{d}} \equiv \sigma^{p \bar{c} \bar{d}} D_{p} .
$$

Acting on a scalar, $\mathcal{T}_{L}=t_{L}$ and $\mathcal{T}_{R}=t_{R}$, since both just act geometrically. But they differ in acting on fields that carry spinor or vector indices. For example, on spinor indices,

$$
t_{L}^{a b} V_{e}=\mathcal{T}_{L}^{a b} V_{e}+\frac{1}{2} \delta_{e}^{a} \delta^{b c} V_{c}-\frac{1}{2} \delta_{e}^{b} \delta^{a c} V_{c} .
$$

For $A d S_{3} \times S^{3}$ we can write the Riemann tensor and the metric tensor as

$$
\begin{aligned}
\bar{R}_{m n p \tau} & =\frac{1}{4}\left(\bar{g}_{m \tau} \bar{R}_{n p}+\bar{g}_{n p} \bar{R}_{m \tau}-\bar{g}_{n \tau} \bar{R}_{m p}-\bar{g}_{m p} \bar{R}_{n \tau}\right) \\
\bar{g}_{m n} & =\frac{1}{2} \sigma_{m}^{a b} \sigma_{n a b} .
\end{aligned}
$$

The sigma matrices $\sigma^{m a b}$ satisfy the algebra $\sigma^{m a b} \sigma_{a c}^{n}+\sigma^{n a b} \sigma_{a c}^{m}=\eta^{m n} \delta_{c}^{b}$ in flat space, where $\eta^{m n}$ is the six-dimensional Minkowski metric. Sigma matrices with lowered indices are defined by $\sigma_{a b}^{m}=\frac{1}{2} \epsilon_{a b c d} \sigma^{m c d}$, although for other quantities indices are raised and lowered with $\delta^{a b}$, so we distinguish $\sigma_{a b}^{m}$ from $\delta_{a c} \delta_{b d} \sigma^{m c d}$. In curved space, $\eta_{m n}$ is replaced by the $A d S_{3} \times S^{3}$ metric $\bar{g}_{m n}$.

We then find from the string constraints that the six-dimensional metric field $g_{r s}$, the dilaton $\phi$, and the two-form $b_{r s}$ satisfy

$$
\begin{aligned}
\frac{1}{2} D^{p} D_{p} b_{r s} & =-\frac{1}{2}\left(\sigma_{r} \sigma^{p} \sigma^{q}\right)_{a b} \delta^{a b} D_{p}\left[g_{q s}+\bar{g}_{q s} \phi\right]+\frac{1}{2}\left(\sigma_{s} \sigma^{p} \sigma^{q}\right)_{a b} \delta^{a b} D_{p}\left[g_{q r}+\bar{g}_{q r} \phi\right] \\
& -\bar{R}_{\tau r s \lambda} b^{\tau \lambda}-\frac{1}{2} \bar{R}_{r}^{\tau} b_{\tau s}-\frac{1}{2} \bar{R}_{s}^{\tau} b_{r \tau} \\
& +\frac{1}{4} F_{\text {asy }}^{++g h} \sigma_{r}^{a b} \sigma_{s}^{e f} \delta_{a h} \delta_{b e} \delta_{g f} \\
\frac{1}{2} D^{p} D_{p}\left(g_{r s}+\bar{g}_{r s} \phi\right) & =-\frac{1}{2}\left(\sigma_{r} \sigma^{p} \sigma^{q}\right)_{a b} \delta^{a b} D_{p} b_{q s}+\frac{1}{2}\left(\sigma_{s} \sigma^{p} \sigma^{q}\right)_{a b} \delta^{a b} D_{p} b_{r q} \\
& -\bar{R}_{\tau r s \lambda}\left(g^{\tau \lambda}+\bar{g}^{\tau \lambda} \phi\right)-\frac{1}{2} \bar{R}_{r}^{\tau}\left(g_{\tau s}+\bar{g}_{\tau s} \phi\right)-\frac{1}{2} \bar{R}_{s}^{\tau}\left(g_{r \tau}+\bar{g}_{r \tau} \phi\right) \\
& +\frac{1}{4} F_{\mathrm{sym}}^{++g h} \sigma_{r g a} \sigma_{s h b} \delta^{a b} .
\end{aligned}
$$


This is the curved space version of the flat space zero Laplacian condition $\partial^{p} \partial_{p} b_{r s}=$ $\partial^{p} \partial_{p} g_{r s}=\partial^{p} \partial_{p} \phi=0$.

Four self-dual tensor and scalar pairs come from the string bispinor fields $F^{++a b}, V_{a b}^{--},, A_{b}^{+-a}, A_{a}^{-+b}$. From the string constraint equations they satisfy

$$
\begin{gathered}
\sigma_{d a}^{p} D_{p} F_{\text {asy }}^{++a b}=0 \\
\frac{1}{4}\left[\delta^{B a} \sigma_{g a}^{r} D_{r} F_{\mathrm{sym}}^{++g H}-\delta^{H a} \sigma_{g a}^{r} D_{r} F_{\mathrm{sym}}^{++g B}\right]=-\frac{1}{4} \epsilon^{B H}{ }_{c d} F_{\text {asy }}^{++c d}
\end{gathered}
$$

We also find

$$
\begin{aligned}
& \frac{1}{2} D^{p} D_{p} V_{c d}^{--}-\frac{1}{2} \delta^{g h} \sigma_{c h}^{p} D_{p} V_{g d}^{--}+\frac{1}{2} \delta^{g h} \sigma_{d h}^{p} D_{p} V_{c g}^{--}+\frac{1}{4} \epsilon_{c d}^{g h} V_{g h}^{--} \\
= & -4 \sigma_{c e}^{m} \sigma_{d f}^{n} \delta^{e f} G_{m n} .
\end{aligned}
$$

The last constraints can be written as

$$
\begin{aligned}
& \epsilon_{\text {eacd }} \quad t_{L}^{c d} h_{b}{ }^{\bar{a}} F^{+-a b}=0 \quad \epsilon_{\bar{e} \bar{b} \bar{c} \bar{d}} t_{R}^{\bar{c} \bar{d}} h_{\bar{a}}^{a} F^{+-\bar{a} \bar{b}}=0 \\
& \epsilon_{\text {eacd }} \quad t_{L}^{c d} h_{b}^{\bar{a}} F^{-+a b}=0, \quad \epsilon_{\bar{e} \bar{b} \bar{c} \bar{d}} t_{R}^{\bar{c} \bar{d}} h_{\bar{a}}^{a} F^{-+\bar{a} \bar{b}}=0
\end{aligned}
$$

where

$$
\begin{aligned}
F^{+-a \bar{a}} & \equiv \delta^{\bar{a} \bar{b}} A_{\bar{b}}^{+-a}+t_{R}^{\bar{a} \bar{b}} A_{\bar{b}}^{+-a} \\
F^{-+a \bar{a}} & \equiv \delta^{a b} A_{b}^{-+\bar{a}}+t_{L}^{a b} A_{b}^{-+\bar{a}}
\end{aligned}
$$

so $F^{+-a b}$ and $F^{-+a b}$ satisfy equations similar to those for $F^{++a b}$.

Independent conditions on the fermion fields are

$$
\begin{aligned}
& \square h_{a}^{\bar{g}} \sigma_{\bar{a} \bar{b}}^{m} \xi_{m \bar{g}}^{-}=-\sigma_{\bar{g} \bar{h}}^{m} \epsilon_{\bar{e} \bar{d} \bar{a} \bar{b}} h_{a}^{\bar{h}} \delta^{\bar{g} \bar{d}} \bar{\chi}_{m}^{+\bar{e}} \\
& \square h_{\bar{a}}^{g} \sigma_{a b}^{m} \bar{\xi}_{m g}^{-}=-\sigma_{g h}^{m} \epsilon_{e d a b} h_{\bar{a}}^{h} \delta^{g d} \chi_{m}^{+e} \\
& t_{L}^{a b} h_{\bar{a}}^{g} \sigma_{a b}^{m} \bar{\xi}_{m g}^{-}=0, \quad t_{R}^{\bar{a} \bar{b}} h_{a}^{\bar{g}} \sigma_{\bar{a} \bar{b}}^{m} \xi_{m \bar{g}}^{-}=0 \\
& t_{L}^{a b} \sigma_{a b}^{m} h_{g}^{\bar{a}} \bar{\chi}_{m}^{+g}=0, \quad t_{R}^{\bar{a} \bar{b}} \sigma_{\bar{a} \bar{b}}^{m} h_{\bar{g}}^{a} \chi_{m}^{+\bar{g}}=0 \\
& \epsilon_{\text {deab }} t_{L}^{a b} h_{\bar{a}}^{g} h_{\bar{b}}^{h} \sigma_{g h}^{m} \chi_{m}^{+e}=0, \quad \epsilon_{\bar{d} \bar{a} \bar{b} \bar{b}} t_{R}^{\bar{a} \bar{b}} h_{a}^{\bar{g}} h_{b}^{\bar{h}} \sigma_{\bar{g} \bar{h}}^{m} \bar{\chi}_{m}^{+\bar{e}}=0 .
\end{aligned}
$$

\section{Comparison With Linearized $A d S$ Supergravity Equations}

We now show that the $A d S_{3} \times S^{3}$ supersymmetric vertex operator constraint equations are equivalent to the linearized supergravity equations for the supergravity multiplet and one tensor multiplet of $D=6, N=(2,0)$ supergravity $^{4}$ expanded around the $A d S_{3} \times S^{3}$ metric and a self-dual three-form. We give the identification of the string vertex operator components in terms of the supergravity fields. 
We will see that the two-form $b_{m n}$ is a linear combination of all the oscillations corresponding to the five self-dual tensor fields and the anti-self-dual tensor field, including the oscillation with non-vanishing background. In flat space, $b_{m n}$ corresponds to a state in the Neveu-Schwarz sector. In our curved space case, the string model describes vertex operators for $A d S_{3}$ background with Ramond-Ramond flux. When matching the vertex operator component fields with the supergravity oscillations, we find that not only the bispinor $V_{a b}^{--}$(which is a Ramond-Ramond field in the flat space case), but also the tensor $b_{m n}$ include supergravity oscillations with non-vanishing self-dual background.

The linearized supergravity equations are given by

$$
\begin{gathered}
D^{p} D_{p} \phi^{i}=\frac{2}{3} \bar{H}_{p r s}^{i} g^{6 p r s} \\
\frac{1}{2} D^{p} D_{p} \quad h_{r s}-\bar{R}_{\tau r s \lambda} h^{\tau \lambda}+\frac{1}{2} \bar{R}_{r}^{\tau} h_{\tau s}+\frac{1}{2} \bar{R}_{s}^{\tau} h_{\tau r}-\frac{1}{2} D_{s} D^{p} h_{p r}-\frac{1}{2} D_{s} D^{p} h_{p r}+\frac{1}{2} D_{r} D_{s} h_{p}^{p} \\
=\quad-\bar{H}_{r}^{i p q} g_{s p q}^{i}-\bar{H}_{s}^{i}{ }^{p q} g_{r p q}^{i}+2 h^{p t} \bar{H}_{r p}^{i q} \bar{H}_{s t q}^{i} \\
D^{p} H_{p r s}=-2 \bar{H}_{p r s}^{i} D^{p} \phi^{i} \\
\quad+\quad B^{i}\left[-\bar{H}_{r}^{i p q} D_{p} h_{q s}+\bar{H}_{s}^{i p q} D_{p} h_{q r}+\bar{H}_{r s}^{i q} D^{p} h_{p q}-\frac{1}{2} \bar{H}_{r s}^{i q} D_{q} h_{p}^{p}\right]
\end{gathered}
$$

where we have defined $H_{p r s} \equiv g_{p r s}^{6}+B^{i} g_{p r s}^{i}$ as a combination of the supergravity exact forms $g^{6} \equiv d b^{6}, g^{i} \equiv d b^{i}$, since we will equate this with the string field strength $H=d b$. We will choose $B^{1}=2$. In zeroeth order, the equations are $\bar{R}_{r s}=-\bar{H}_{r p q}^{i} \bar{H}_{s}^{i p q}$.

We define the vertex operator components in terms of the supergravity fields $g_{p r s}^{i}, g_{p r s}^{6}, h_{r s}, \phi^{i}, 1 \leq i \leq 5$, (and $\left.2 \leq I \leq 5\right)$ as

$$
\begin{gathered}
H_{p r s} \equiv g_{p r s}^{6}+2 g_{p r s}^{1}+B^{I} g_{p r s}^{I} \\
g_{r s} \equiv h_{r s}-\frac{1}{6} \bar{g}_{r s} h_{\lambda}^{\lambda} \\
\phi=-\frac{1}{3} h_{\lambda}^{\lambda} \\
F_{\mathrm{sym}}^{++a b}=\frac{2}{3}\left(\sigma_{p} \sigma_{r} \sigma_{s}\right)^{a b} B^{I} g_{p r s}^{I}+\delta^{a b} \phi^{++} \\
F_{\text {asy }}^{++a b}=\sigma^{a b} D_{p} \phi^{++} \\
\phi^{++}=4 C^{I} \phi^{I}
\end{gathered}
$$

which follows from choosing the graviton trace $h_{\lambda}^{\lambda}$ to satisfy $\phi^{1}-h_{\lambda}^{\lambda} \equiv-2 C^{I} \phi^{I}$, and we have used $H_{p r s} \equiv \partial_{p} b_{r s}+\partial_{r} b_{s p}+\partial_{s} b_{p r}$.

The combinations $C^{I} \phi^{I}$ and $B^{I} g_{\text {prs }}^{I}$ reflect the $S O(4)_{\mathrm{R}}$ symmetry of the $D=$ $6, N=(2,0)$ theory on $A d S_{3} \times S^{3}$. We relabel $C^{I}=C_{++}^{I}, B^{I}=B_{++}^{I}$. To define the 
remaining string components in terms of supergravity fields, we consider linearly independent quantities $C_{\ell}^{I} \phi^{I}, B_{\ell}^{I} g_{p r s}^{I}, \ell=++,+-,-+,--$.

$$
\begin{aligned}
F_{\mathrm{sym}}^{+-a b} & =\frac{2}{3}\left(\sigma_{p} \sigma_{r} \sigma_{s}\right)^{a b} B_{+-}^{I} g_{p r s}^{I}+\delta^{a b} \phi^{+-} \\
F_{\mathrm{asy}}^{+-a b} & =\sigma^{p a b} D_{p} \phi^{+-} \\
\phi^{+-} & =4 C_{+-}^{I} \phi^{I} \\
F_{\mathrm{sym}}^{-+a b} & =\frac{2}{3}\left(\sigma_{p} \sigma_{r} \sigma_{s}\right)^{a b} B_{-+}^{I} g_{p r s}^{I}+\delta^{a b} \phi^{-+} \\
F_{\mathrm{asy}}^{-+a b} & =\sigma^{p a b} D_{p} \phi^{-+} \\
\phi^{-+} & =4 C_{-+}^{I} \phi^{I} .
\end{aligned}
$$

$V_{a b}^{--}$is given in terms of the fourth tensor/scalar pair $C_{--}^{I} \phi^{I}, B_{--}^{I} g_{m n p}^{I}$ through

$D^{p} D_{p} V_{c d}^{--}-\delta^{g h} \sigma_{c h}^{p} D_{p} V_{g d}^{--}+\delta^{g h} \sigma_{d h}^{p} D_{p} V_{c g}^{--}+\frac{1}{2} \epsilon_{c d}^{g h} V_{g h}^{--}=-8 \sigma_{c e}^{m} \sigma_{d f}^{n} \delta^{e f} G_{m n}$.

These field definitions allow us to identify the string constraint equations for the $A d S_{3}$ vertex operators as precisely those which require the vertex operator field components to satisfy the linearized supergravity equations reviewed in this section.

Likewise, the fermion constraints imply the linearized AdS supergravity equations for the gravitinos and spinors, due to the above correspondence for the bosons and the supersymmetry of the two theories.

\section{Acknowledgements}

This was supported in part by the U.S. Department of Energy, Grant No. DE-FG 05-85ER40219/Task A. It reviews work done in collaboration with E. Witten.

\section{References}

1. N. Berkovits, C. Vafa, and E. Witten, "Conformal Field Theory of AdS Background With Ramond-Ramond Flux", JHEP 9903 (1999) 018, hep-th/9902098.

2. L. Dolan and E. Witten, "Vertex Operators for AdS3 Background With RamondRamond Flux", JHEP 9911 (1999) 003, hep-th/9910205.

3. M. Bershadsky, S. Zhukov, and A. Vaintrob, "PSL(N/N) Sigma Model As A Conformal Field Theory", Nucl. Phys. B559 (1999) 205, hep-th/9902180.

4. L. Romans, "Self-Duality for Interacting Fields", Nucl. Phys. B276 (1986) 71.

5. N. Berkovits, "Super-Poincare Covariant Quantization of the Superstring", JHEP 0004 (2000) 018, hep-th/0001035.

6. N. Berkovits and O. Chandia, "Superstring Vertex Operators in an $A d S_{5}$ Background", hep-th/0009168 